\title{
IBRUTINIB IN THE MANAGEMENT OF NON-HODGKIN'S LYMPHOMA: A CASE REPORT
}

\author{
SREE DURGA TS ${ }^{1}$, PAVITHRAN $K^{2}$, UMA DEVI P* ${ }^{3 *}$
}

${ }^{1}$ Department of Pharmacy Practice, Amrita School of Pharmacy, Amrita Vishwa Vidyapeetham, Kochi, Kerala, India. ${ }^{2}$ Department of Medical Oncology and Hematology, Amrita Institute of Medical Sciences and Research Centre, Amrita Vishwa Vidyapeetham, Kochi, Kerala, India. ${ }^{3}$ Department of Pharmacology, Amrita School of Pharmacy, Amrita Vishwa Vidyapeetham, Kochi, Kerala, India.

Email: umadevip@aims.amrita.edu/umadeviaims@gmail.com

Received: 24 February 2018, Revised and Accepted: 31 July 2018

\section{ABSTRACT}

Non-Hodgkin's lymphoma (NHL) is a diverse group of lymphoid neoplasms, the prevalence of which has increased over the past few decades. NHL is diverse in the manner of presentation, response to various treatment and prognosis. The current case report describes a 40 -year-old man who was diagnosed with small lymphocytic lymphoma/chronic lymphocytic leukemia in 2006. The patient had disease progression during the course of 10 years from the time of diagnosis for which he received multiple lines of chemotherapy (chlorambucil/prednisolone; rituximab/cyclophosphamide/ fludarabine; bendamustine/rituximab; and ofatumumab). However, in 2016, his disease again showed signs of progression, and hence he was started on ibrutinib $140 \mathrm{mg} 3$ times daily. After treatment with ibrutinib, there were no clinical nodes and hepatosplenomegaly, and all counts also normalized. Since the commencement of this agent, no disease progression was observed for almost 16 months. However, in July 2017, again disease progression occurred, and the patient was started on with cyclophosphamide, vincristine, and prednisone (COP) regimen. He received one cycle of COP regimen and continued on treatment with ibrutinib, and the treatment was well tolerated. In December 2017, he expired due to the progression of the disease. Ibrutinib, a Bruton's tyrosine kinase inhibitor, appears to be safe and effective in providing long-term disease control even in refractory cases of NHL.

Keywords: Non-Hodgkin's lymphoma, Ibrutinib, Bruton's tyrosine kinase.

(C) 2018 The Authors. Published by Innovare Academic Sciences Pvt Ltd. This is an open access article under the CC BY license (http://creativecommons. org/licenses/by/4. 0/) DOI: http://dx.doi.org/10.22159/ajpcr.2018.v11i12.27983

\section{INTRODUCTION}

Non-Hodgkin's lymphomas (NHLs) consist of a heterogeneous group of malignancies arising from lymphoid tissue, with varied clinical, pathological, and biological features. In India, incidence rates of 2.9 and 1.5 per 100,000 have been reported in men and women, respectively [1-3]. Chronic lymphocytic leukemia (CLL) often grouped as NHL, is a lymphoproliferative disease that has a highly variable clinical course and may involve the bone marrow, blood, and solid lymphoid organs [4].

The therapeutic options for the management of CLL have advanced over the years from chemotherapy with chlorambucil, to chemoimmunotherapy with cyclophosphamide, doxorubicin, vincristine, prednisone, fludarabine, and rituximab [5]. However, in many patients, the presence of comorbidities, low bone marrow reserve, acquired genetic mutations make the treatment suboptimal and resulting in relapse [6]. During the recent years, research has focused on drugs targeting novel mechanisms such as Bruton's tyrosine kinase and apoptosis proteins [4]

Ibrutinib is an orally active, irreversible covalent inhibitor of Bruton's tyrosine kinase, a signaling molecule of the B-cell receptor pathway. Studies have demonstrated that ibrutinib prevents the activation of downstream pathways affected by Bruton's tyrosine kinase and regulates adhesion, homing, proliferation, differentiation, and survival of cancerous cells [7,8]. It is Food and Drug Administration approved for CLL patients who have received at least one prior therapy [9]. The current report presents the case of a patient treated with ibrutinib at our center.

\section{CASE REPORT}

A 40-year-old male, with a history of swelling in the right neck since 3 years was admitted to our institution in July 2006. On local examination, multiple small swelling in the right and left side of the neck in the anterior and posterior triangle was observed with the largest measuring about $3 \times 2 \mathrm{~cm}$. Abdominal ultrasound examination revealed multiple para-aortic, mesenteric and iliac lymph nodes, and largest measuring $43 \times 17 \mathrm{~mm}$. Excision biopsy of the lymph node revealed small lymphocytic lymphoma/CLL. The patient received chemotherapy with chlorambucil and prednisolone for 2 weeks. In 2011, the patient had disease progression and was treated with six cycles of chemotherapy with rituximab/cyclophosphamide/ fludarabine, and had a progression-free survival (PFS) of 18 months. In November 2013, again the disease got worsened, and large neck nodes were evident. Hence, the patient received six cycles of chemotherapy with R-bendamustine and rituximab. In July 2015, the patient presented with complaints of low back pain, decreased urine output, abdominal distension, and constipation. His peripheral smear revealed normocytic normochromic anemia with thrombocytopenia with $72 \%$ mature lymphocytes. On magnetic resonance imaging (MRI), marrow infiltration of spine, and multiple significantly enlarged discrete and conglomerate intra-abdominal nodal masses were evident. The patient was treated with cyclophosphamide as a bridge to ofatumumab therapy. Following this, the patient received weekly ofatumumab therapy for 8 weeks without any major complaints until October 2015. However, in February 2016, the patient presented with complaints of breathlessness, hiccough, abdominal discomfort, and generalized weakness. Ultrasonography of abdomen showed multiple discrete and conglomerate hypoechoic nodes in the periportal, peripancreatic, para-aortic, splenic hilum, and along common iliac vessels. Hepatosplenomegaly was also seen. His hemoglobin level was $8.6 \mathrm{~g} / \mathrm{dl}$; platelet count was $51 \mathrm{ku} / \mathrm{ml}$, and total count was $74.2 \mathrm{ku} / \mathrm{ml}$. In view of progression, the patient was started on ibrutinib $140 \mathrm{mg}$ thrice daily from March 2016. After treatment with ibrutinib, there were no clinical nodes and hepatosplenomegaly; and all counts also normalized (hemoglobin level: $13.1 \mathrm{~g} / \mathrm{dl}$, platelet count: $184 \mathrm{ku} / \mathrm{ml}$, and total count: $113 \mathrm{ku} / \mathrm{ml}$ ). In March 2017, he had cough with a minimal expectoration and breathlessness. In July 2017, he complained of leg pain. MRI showed marrow involvement of cervical, 
thoracic and lumbar vertebra with epidurals of tissue component at D6 and D9 level causing compression of spinal cord; enlarged paraaortic and iliac nodes and diffuse marrow involvement of pelvic bones and bilateral femurs with soft tissue deposits along the iliac crests. In view of the disease progression, the patient was started on with cyclophosphamide/vincristine/prednisone. In September 2017, he presented with complaints of pneumonia for which he was admitted and treated conservatively. In December 2017, he presented with complaints of breathlessness and expired due to progressive disease.

\section{DISCUSSION}

Lymphoma is a common hematologic malignancy. Survival rates for NHL have significantly improved over the past several decades, but patients with relapsed and refractory disease continue to do poorly. The prognosis is poor for most patients even with most current treatment regimens.

The choice of treatment depends on the patient's age, comorbidities, and tolerance to chemotherapeutic agents. Ibrutinib is a recently approved agent that targets the B cell receptor pathway that has an important role in B cell maturation, proliferation, and survival. In a comparative phase 3 trial of ibrutinib and ofatumumab, ibrutinib at a daily dosage of $420 \mathrm{mg}$ demonstrated higher overall response rate (43\% vs. $4 \%$ ) and improved PFS at 12 months (90\% vs. 81\%) [10]. In our patient, ibrutinib monotherapy produced a PFS of almost 16 months. This appears promising since our patient had an advanced disease profile and had received treatment with chlorambucil/prednisolone; rituximab/cyclophosphamide/fludarabine; bendamustine/rituximab; and ofatumumab before therapy with ibrutinib. Hence, if ibrutinib is started at an earlier stage of the disease, the better outcome can be expected.

In clinical studies, ibrutinib has been found to be well tolerated. The most common adverse events associated with ibrutinib include diarrhea, fatigue, cough, nausea, and upper respiratory infection. Serious adverse effects usually occur infrequently and include neutropenia, diarrhea, hypertension, fatigue, and pneumonia [11]. The patient we represented in this report had experienced episodes of respiratory infection, cough, breathlessness, and pneumonia during the therapy. These adverse effects may possibly be due to ibrutinib therapy.

One of the drawbacks of ibrutinib therapy is that it does not achieve minimal residual disease negative complete remissions and therefore in the majority of patients, must be given indefinitely if relapse is to be prevented. This can lead to a considerable cost to the patient and can also result in risk of toxicity such as cardiac arrhythmias, hypertension, and bleeding [12].

Combination of ibrutinib with other therapy such as rituximab, ofatumumab, ublituximab, and obinutuzumab is being explored. Our patient had received monotherapy with ibrutinib. In future studies, one needs to explore whether ibrutinib monotherapy or combination therapy is ideal for CLL patients. The duration of ibrutinib therapy to get optimum results also needs to be explored in future studies.

\section{CONCLUSION}

Ibrutinib, a Bruton's tyrosine kinase inhibitor, appears to be safe and very effective in providing long-term disease control even in refractory cases of NHL. Since the data we represented here was only from a single patient, further studies with more numbers can reveal a better perspective regarding the safety and efficacy of ibrutinib in the management of NHL in the Indian population.

\section{AUTHOR'S CONTRIBUTION}

Sree Durga TS was involved in collecting data and preparing the initial draft of the manuscript.

Pavithran K and Uma Devi P were involved in critical revision of the manuscript for intellectual content.

\section{CONFLICTS OF INTEREST}

The authors declare that they have no conflicts of interest.

\section{REFERENCES}

1. Nair R, Arora N, Mallath MK. Epidemiology of non-hodgkin's lymphoma in India. Oncology 2016;91 Suppl 1:18-25.

2. Nair IR, Jojo A, Keechilat P. Pathological profile of lymphomas in a tertiary care centre in Kerala. Pathol Int 2012;62:360-2.

3. Gupta M, Dahiya J, Marwaha RK, Dureja H. Therapies in cancer treatment: An overview. Int J Pharm Pharm Sci 2015;7:1-9.

4. Gomes LC, Ferrão ALM, Evangelista FCG, de Almeida TD, Barbosa RC, Carvalho MDG, et al. Advances in chronic lymphocytic leukemia pharmacotherapy. Biomed Pharmacother 2018;97:349-58.

5. Ahmad A, Ali MD, Almahmoud S. Safety and efficacy of three treatment regimens of R-CHOP, E-CHOP, and R-CHOEP in non-hodgkin's lymphoma cancer patients. Asian J Pharm Clin Res 2018;11:238-41.

6. Shustik C, Bence-Bruckler I, Delage R, Owen CJ, Toze CL, Coutre S, et al. Advances in the treatment of relapsed/refractory chronic lymphocytic leukemia. Ann Hematol 2017;96:1185-96.

7. Raedler LA. Imbruvica (Ibrutinib): First drug approved for the treatment of patients with waldenström's macroglobulinemia. Am Health Drug Benefits 2016;9:89-92.

8. Gayko U, Fung M, Clow F, Sun S, Faust E, Price S, et al. Development of the bruton's tyrosine kinase inhibitor ibrutinib for B cell malignancies. Ann N Y Acad Sci 2015;1358:82-94

9. US Prescribing Information of IMBRUVICA (Ibrutinib) Capsules. Available from: https://www.accessdata.fda.gov/drugsatfda docs/ label/2015/205552s002lbl.pdf. [Last retrieved on 2018 Apr 7]

10. Lee CS, Rattu MA, Kim SS. A review of a novel, bruton's tyrosine kinase inhibitor, ibrutinib. J Oncol Pharm Pract 2016;22:92-104

11. Vela CM, McBride A, Jaglowski SM, Andritsos LA. Ibrutinib for treatment of chronic lymphocytic leukemia. Am J Health Syst Pharm 2016;73:367-75.

12. Thompson PA, Burger JA. Bruton's tyrosine kinase inhibitors: First and second generation agents for patients with chronic lymphocytic leukemia (CLL). Expert Opin Investig Drugs 2018;27:31-42. 